\title{
Models and Algorithms for Fuzzy Multi-objective Multi-follower Linear Bilevel Programming in a Partial Cooperative Situation
}

\author{
Guangquan Zhang ${ }^{1}$ Jie Lu$^{1}$ Xianyi Zeng ${ }^{2}$ \\ ${ }^{1}$ Faculty of Information Technology, University of Technology, Sydney \\ PO Box 123, Broadway, NSW 2007, Australia \\ ${ }^{2}$ Ecole Nationale Supérieure des Arts et Industries Textiles, 9 rue de l'Ermitage 59100 Roubaix, France
}

\begin{abstract}
Basic bilevel programming deals with hierarchical optimization problems in which the leader at the upper level attempts to optimize his/her objective, subject to a set of constraints and his/her follower's solution, and the follower at the lower level tries to find an optimized strategy according to each of possible decisions made by the leader. Three issues may be involved in a basic bilevel decision problem. One is that bilevel decision making model may involve uncertain parameters which appear either in the objective functions or constraints of the leader or the follower or both. Second, the leader and the follower may have multiple conflict objectives that should be optimized simultaneously. Third, there may have multiple followers and partial shared their decision variables among followers in a real decision situation. Following our previous work, this study proposes a set of fuzzy multi-objective multi-follower linear bilevel programming models to describe the three issues. It also develops an approximation branch-and-bound algorithm to solve such kinds of problems.
\end{abstract}

Keywords: Bilevel programming, Branch and bound algorithm, Fuzzy sets, Optimization

\section{Introduction}

Bilevel programming (BP) arises where decisions are made in a two level hierarchical order and each decision maker has no direct control upon the decision of the other, but actions taken by one decision maker effect returns from the other. It is developed for mainly solving decentralized planning problems $[1,2$, $4-6,18,19]$. Decision maker at the upper level is termed as the leader, and at the lower level, the follower. The leader and the follower each tries to optimize his/her own objective function, but the decision affects the objective value of the other level [12].
The majority of research on BP has centered on the linear version of the problem, i.e., linear BP problems. A set of approaches and algorithms have been well developed such as well known Kuhn-Tucker approach [2], Kth-best approach [3] and branch-andbound algorithm [2, 7]. In general, there are two fundamental issues in BP theory and practice. One is how to model a real-world bilevel programming problem, and the other is how to find a solution for the problem. Although much research has been done in the area, existing results cannot adequately model and well solve a bilevel problem when it corresponds to the following situations.

First, the upper level or the lower level of a bilevel decision may have multiple conflicting objectives which should be considered simultaneously by the leader or the follower. For example, a coordinator of a multi-division firm considers three objectives in making an aggregate production plan: maximise net profits, maximise quality of products, and maximise worker satisfaction. The three objectives could be in conflict with each other, but must be considered simultaneously. Any improvement in one objective may be achieved only at the expense of others. One level multi-objective decision-making problem has been well researched and many other researchers. But in a bilevel model, selection of an alternative solution for the leader is affected by his/her followers' optimal reactions. Therefore, how to find a solution for the leader which has multiple objectives under consideration of both its constraints and its followers' decision is a new issue.

Second, existing BP approaches mainly suppose the situation in which the objective functions and the constraints of the leader and the follower are characterized with precise parameters. Therefore, the parameters are required to be fixed at the some values in an experimental and/or subjective manner through the experts' understanding of the nature of the parameters in the problem-formulation process. It has been observed that, in most real-world situations, for example, power market and business management, the possible values of these parameters are often only 
imprecisely or ambiguously known to the experts who establish this model. With this observation, it would be certainly more appropriate to interpret the experts' understanding of the parameters as fuzzy numerical data which can be represented by means of fuzzy sets [20]. A BP problem in which the parameters, either in objective functions or in constrains of the leader or the follower, are described by fuzzy values is called a fuzzy BP problem in the study. The fuzzy BP problem was first researched by Sakawa et al. [13, 14]. Sakawa et al. formulated cooperative fuzzy BP problem and proposed a fuzzy bilevel programming approach for solving the problem where Sakawa introduced the concepts of $\alpha$-bilevel programming based on the basis of fuzzy number $\alpha$-level sets. The third, multiple followers may be involved and the leader's decision will be affected not only by those followers' individual reactions but also by the relationships among these followers. As uncertain information could occur in the objectives and the constraints of both the leader and his/her multiple followers, the problem becomes very complex.

This paper deals with the three situations together. As the relationship among followers can be with many situations which need different models and algorithms, this paper focuses on the situation where all followers shard their decision variables. Based on the extended solution concept and related theorems of BP [8, 9, 1517], we have solved fuzzy BP problems [21-25]. We then conducted research on the model and solution where the leader and the follower to have multiple objectives with fuzzy parameters [11, 26-28]. This paper extends our previous models by allowing multiple followers with partial shared their decision variables, called a fuzzy multi-objective multifollower linear bilevel programming with partial shared decision variables (FMMBP-PC) problem. It also develops an approximation branch-and-bound algorithm to solve the general FMBP-PC problem.

Following the introduction, Section 2 gives FMBP-PC models, related definitions, theorems and properties [10, 26]. A general fuzzy number based approximation branch-and-bound algorithm for solving FMMBP-PC problems is presented in Section 3. Conclusions are discussed in Section 4.

\section{Fuzzy Multi-objective Multi- follower Linear Bilevel Programming with Partial Shared Variables Model}

Let $R$ be the set of all real numbers, $R^{n}$ be $n$ dimensional Euclidean space, and $x=\left(x_{1}, x_{2}, \ldots, x_{n}\right)^{T}$, $y=\left(y_{1}, y_{2}, \ldots, y_{n}\right)^{T} \in R^{n}$ be any two vectors, where $x_{i}$, $y_{i} \in R, i=1,2, \ldots, n$ and $T$ denotes the transpose of the vector. For any two vectors $x, y \in R^{n}$, we write $x \geq y$ iff $x_{i} \geq y_{i}, \forall i=1,2, \cdots, n ; \quad x \geq y$ iff $x \geq y$ and

$x \neq y ; x>y$ iff $x_{i}>y_{i}, \forall i=1,2, \ldots, n$.

Let $F(R)$ be the set of all finite fuzzy numbers. By the decomposition theorem of fuzzy sets, we have

$$
\tilde{a}=\bigcup_{\lambda \in[0,1]} \lambda\left[a_{\lambda}^{L}, a_{\lambda}^{R}\right],
$$

for every $\tilde{a} \in F(R)$.

Definition 2.1 Let $\tilde{a}_{i} \in F(R), i=1,2, \cdots, n$. We define $\quad \tilde{a}=\left(\tilde{a}_{1}, \tilde{a}_{2}, \cdots, \tilde{a}_{n}\right): \quad \mu_{\tilde{a}}: R^{n} \rightarrow \quad\left[\begin{array}{ll}0, & 1\end{array}\right]$, $x \mapsto \wedge_{i=1}^{n} \mu_{\tilde{a}_{i}}\left(x_{i}\right)$, where $x=\left(x_{1}, x_{2}, \ldots, x_{n}\right)^{T} \in R^{n}$, and $\tilde{a}$ is called an $n$-dimensional fuzzy number on $R^{n}$. If $\tilde{a}_{i} \in F^{*}(R), \quad i=1,2, \cdots, n, \quad \tilde{a}$ is called an $n$ dimensional finite fuzzy number on $R^{n}$.

Let $F\left(R^{n}\right)$ be the set of all $n$-dimensional fuzzy numbers and the set of all $n$-dimensional finite fuzzy numbers on $R^{n}$ respectively.

Definition 2.2 For any $n$-dimensional fuzzy numbers $\tilde{a}, \tilde{b} \in F\left(R^{n}\right)$, we define

1. $\tilde{a} \succeq \tilde{b}$ iff $a_{\lambda}^{L} \geq b_{\lambda}^{L}$ and $a_{\lambda}^{R} \geq b_{\lambda}^{R}, \lambda \in(0,1]$;

2. $\tilde{a} \succeq \tilde{b}$ iff $a_{\lambda}^{L} \geq b_{\lambda}^{L}$ and $a_{\lambda}^{R} \geq b_{\lambda}^{R}, \lambda \in(0,1]$;

3. $\tilde{a} \succ \tilde{b}$ iff $a_{\lambda}^{L}>b_{\lambda}^{L}$ and $a_{\lambda}^{R}>b_{\lambda}^{R}, \lambda \in(0,1]$.

We call the binary relations $\succeq$, $\succeq$ and $\succ$ a fuzzy max order, a strict fuzzy max order and a strong fuzzy max order, respectively.

Fuzzy multi-objective multi-follower linear bilevel programming with partial shared decision variables problems is the cooperative situation where the followers partial share the decision variables in their objectives and constraints. However, there are four different sub-cases within the cooperative situation which are determined by the relationships among the objectives and constrains of the followers. Each follower may have an individual objective whatever sharing their constraints with other followers. Consider the following FMBP-PC problems:

Model I. A FMMBP-PC problem in which $K(\geq 2)$ followers are involved and there are shared objective functions and constraint functions and partial decision variables among followers is defined as follows:

$$
\begin{aligned}
& \text { For } x \in X \subset R^{n}, y_{i} \in Y_{i} \subset R^{m_{i}}, Y=\left(Y_{1}, \cdots, Y_{k}, Z\right)^{T}, \\
& F: X \times Y_{1} \times \times \times \times Y_{K} \times Z \rightarrow F\left(R^{s}\right), \quad f_{i}: X \times Y_{i} \times Z \rightarrow \\
& F\left(R^{t}\right) \text { and } i=1,2 . \cdots, K .
\end{aligned}
$$

It consists of finding a solution to the upper level problem

$$
\begin{aligned}
\min _{x \in X} F(x, y)=\left(\tilde{c}_{1}^{1} x\right. & +\sum_{j=1}^{K} \tilde{d}_{1 j}^{1} y_{j}+\tilde{d}_{1}^{1} z, \tilde{c}_{2}^{1} x+\sum_{j=1}^{K} \tilde{d}_{2 j}^{1} y_{j} \\
& \left.+\tilde{d}_{2}^{1} z, \cdots, \tilde{c}_{s}^{1} x+\sum_{j=1}^{K} \tilde{d}_{s j}^{1} y_{j}+\tilde{d}_{s}^{1} z\right)^{T}
\end{aligned}
$$




$$
\text { s.t. } \tilde{A}^{1} x+\sum_{j=1}^{k} \tilde{B}_{j}^{1} y_{j}+\widetilde{B}^{1} z \preceq \tilde{b}^{1}
$$

where $y_{i}(i=1,2, \cdots, K)$, for each value of $x$, is the solution of the lower level problem:

$$
\begin{array}{r}
\min _{y, \in Y_{j}, z \in Z} f(x, y)=\left(\tilde{c}_{1}^{2} x+\sum_{j=1}^{K} \tilde{d}_{1 j}^{2} y_{j}+\tilde{d}_{1}^{2} z, \tilde{c}_{2}^{2} x+\sum_{j=1}^{K} \tilde{d}_{2 j}^{2} y_{j}\right. \\
\left.+\tilde{d}_{2}^{2} z, \cdots, \tilde{c}_{t}^{2} x+\sum_{j=1}^{K} \tilde{d}_{t j}^{2} y_{j}+\tilde{d}_{t}^{2} z\right)^{T}
\end{array}
$$

s.t. $\tilde{A}^{2} x+\sum_{j=1}^{K} \tilde{B}_{j}^{2} y_{j}+\tilde{B}^{2} z \preceq \tilde{b}^{2}$

where $\tilde{c}_{i}^{1}, \tilde{c}_{j}^{2} \in F\left(R^{n}\right), \quad \tilde{b}^{1} \in F\left(R^{p}\right), \quad \tilde{b}^{2} \in F\left(R^{q}\right), \quad \tilde{a}_{i j}^{1} \in$ $F(R), \quad \tilde{A}^{1}=\left(\tilde{a}_{i j}^{1}\right)_{p \times n}, \quad \tilde{b}_{i j k}^{1}, \tilde{e}_{i j}^{1} \in F(R), \quad \tilde{B}_{k}^{1}=\left(\tilde{b}_{i j k}^{1}\right)_{p \times m}, \quad \tilde{B}^{1}=$ $\left(\tilde{e}_{i j}^{1}\right)_{p \times m}, \tilde{a}_{i j}^{2} \in F(R), \tilde{A}^{2}=\left(\tilde{a}_{i j}^{2}\right)_{q \times n}, \tilde{d}_{i k}^{1}, \tilde{d}_{i}^{1}, \tilde{d}_{j k}^{2}, \tilde{d}_{j}^{2} \in F\left(R^{m}\right)$, $\tilde{b}_{i j k}^{2}, \tilde{b}_{i j}^{2} \in F(R), \tilde{B}_{k}^{2}=\left(\tilde{b}_{i j k i j}^{2}\right)_{q \times m}, \tilde{B}^{2}=\left(\tilde{b}_{i j}^{2}\right)_{q \times m}, i=1,2, \cdots, s$, $j=1,2, \cdots, t$, and $k=1,2, \cdots, K$.

Model II. A FMMBP-PC problem in which $K(\geq 2)$ followers are involved and there are shared objective functions and partial decision variables but different constraint functions among followers is defined as follows.

For $x \in X \subset R^{n}, y_{i} \in Y_{i} \subset R^{m_{i}}, Y=\left(Y_{1}, \cdots, Y_{k}, Z\right)^{T}$, $F: X \times Y_{1} \times \times \ldots \times Y_{K} \times Z \rightarrow F\left(R^{s}\right), f_{i}: X \times Y_{i} \times Z \rightarrow$ $F\left(R^{t}\right)$ and $i=1,2 \ldots, K$.

It consists of finding a solution to the upper level problem

$$
\begin{aligned}
\min _{x \in X} F(x, y)=\left(\tilde{c}_{1}^{1} x\right. & +\sum_{j=1}^{K} \tilde{d}_{1 j}^{1} y_{j}+\tilde{d}_{1}^{1} z, \tilde{c}_{2}^{1} x+\sum_{j=1}^{K} \tilde{d}_{2 j}^{1} y_{j} \\
& \left.+\tilde{d}_{2}^{1} z, \cdots, \tilde{c}_{s}^{1} x+\sum_{j=1}^{K} \tilde{d}_{1 j}^{1} y_{j}+\tilde{d}_{s}^{1} z\right)^{T}
\end{aligned}
$$

s.t. $\tilde{A}^{1} x+\sum_{j=1}^{k} \tilde{B}_{j}^{1} y_{j}+\widetilde{B}^{1} z \stackrel{\tilde{b}^{1}}{=}$

where $y_{i}(i=1,2, \cdots, K)$, for each value of $x$, is the solution of the lower level problem:

$$
\begin{array}{r}
\min _{y, Y_{J}, z \in Z} f(x, y)=\left(\tilde{c}_{1}^{2} x+\sum_{j=1}^{K} \tilde{d}_{1 j}^{2} y_{j}+\tilde{d}_{1}^{2} z, \tilde{c}_{2}^{2} x+\sum_{j=1}^{K} \tilde{d}_{2 j}^{2} y_{j}\right. \\
\left.+\tilde{d}_{2}^{2} z, \cdots, \tilde{c}_{t}^{2} x+\sum_{j=1}^{K} \tilde{d}_{i j}^{2} y_{j}+\tilde{d}_{t}^{2} z\right)^{T}
\end{array}
$$

s.t. $\tilde{A}_{r}^{2} x+\sum_{j=1}^{K} \widetilde{B}_{r j}^{2} y_{j}+\widetilde{B}_{r}^{2} z \precsim \tilde{b}_{r}^{2}, r=1,2, \cdots, K$,

where $\quad \tilde{c}_{i}^{1}, \tilde{c}_{j}^{2} \in F\left(R^{n}\right), \tilde{b}^{1} \in F\left(R^{p}\right), \tilde{b}^{2} \in F\left(R^{q}\right), \tilde{a}_{i j}^{1} \in$ $F(R), \tilde{A}^{1}=\left(\tilde{a}_{i j}^{1}\right)_{p \times n}, \tilde{b}_{i j k}^{1}, \tilde{e}_{i j}^{1} \in F(R), \widetilde{B}_{k}^{1}=\left(\tilde{b}_{i j k}^{1}\right)_{p \times m}, \widetilde{B}^{1}=$ $\left(\tilde{b}_{i j}^{1}\right)_{p \times m}, \tilde{a}_{i j}^{2 r} \in F(R), \tilde{A}_{r}^{2}=\left(\tilde{a}_{i j}^{2 r}\right)_{q \times n}, \tilde{d}_{i k}^{1}, \tilde{d}_{i}^{1}, \tilde{d}_{j k}^{2}, \tilde{d}_{j}^{2} \in F\left(R^{m}\right)$, $\tilde{b}_{i j k}^{2 r}, \tilde{b}_{i j}^{2 r} \in F(R), \widetilde{B}_{k r}^{2}=\left(\tilde{b}_{i j k}^{2 r}\right)_{q \times m}, \tilde{B}_{r}^{2}=\left(\tilde{b}_{i j}^{2 r}\right)_{q \times m}, i=1,2, \cdots, s$, $j=1,2, \cdots, t$, and $k=1,2, \cdots, K$.
Model III. A FMMBP-PC problem in which $K(\geq 2)$ followers are involved and there are shared partial decision variables and constraint functions but different objective functions among them is defined as follows.

For $x \in X \subset R^{n}, y_{i} \in Y_{i} \subset R^{m_{i}}, Y=\left(Y_{1}, \cdots, Y_{k}, Z\right)^{T}$, $F: X \times Y_{1} \times \times \ldots \times Y_{K} \times Z \rightarrow F\left(R^{s}\right), f_{i}: X \times Y_{i} \times Z \rightarrow$ $F\left(R^{t}\right)$ and $i=1,2 \cdots, K$.

It consists of finding a solution to the upper level problem

$$
\begin{aligned}
& \min _{x \in X} F(x, y)=\left(\tilde{c}_{1}^{1} x+\sum_{j=1}^{K} \tilde{d}_{1 j}^{1} y_{j}+\tilde{d}_{1}^{1} z, \tilde{c}_{2}^{1} x+\sum_{j=1}^{K} \tilde{d}_{2 j}^{1} y_{j}\right. \\
& \left.+\tilde{d}_{2}^{1} z, \cdots, \tilde{c}_{s}^{1} x+\sum_{j=1}^{K} \tilde{d}_{s j}^{1} y_{j}+\tilde{d}_{s}^{1} z\right)^{T}
\end{aligned}
$$

where $y_{i}(i=1,2, \cdots, K)$, for each value of $x$, is the solution of the lower level problem:

$$
\begin{aligned}
\min _{y, j \in J, z \in Z} f_{i}(x, y)=\left(\tilde{c}_{i i}^{2} x\right. & +\sum_{j=1}^{K} \tilde{d}_{i j}^{2} y_{j}+\tilde{d}_{i i}^{2} z, \tilde{c}_{i 2}^{2} x+\sum_{j=1}^{K} \tilde{d}_{i 2}^{2} y_{j} \\
& \left.+\tilde{d}_{i 2}^{2} z, \cdots, \tilde{c}_{i i}^{2} x+\sum_{j=1}^{K} \tilde{d}_{i j}^{2} y_{j}+\tilde{d}_{i t}^{2} z\right)^{T}
\end{aligned}
$$

s.t. $\tilde{A}^{2} x+\sum_{j=1}^{K} \tilde{B}_{j}^{2} y_{j}+\tilde{B}^{2} z \preceq \tilde{b}^{2}$

where $\quad \tilde{c}_{i}^{1}, \tilde{c}_{z j}^{2} \in F\left(R^{n}\right), \tilde{b}^{1} \in F\left(R^{p}\right), \tilde{b}^{2} \in F\left(R^{q}\right), \tilde{a}_{i j}^{1} \in$ $F(R), \tilde{b}_{i j}^{1} \in F(R), \tilde{B}_{z}^{1}=\left(\tilde{b}_{i j}^{1}\right)_{p \times m}, \quad \tilde{e}_{i j} \in F(R), \quad \tilde{A}^{2}=\left(\tilde{e}_{i j}\right)_{q \times n}$, $\tilde{d}_{i z}^{1}, \tilde{d}_{g j z}^{2} \in F\left(R^{m}\right), i=1,2, \cdots, s, j=1,2, \cdots, t$, and $\tilde{B}_{z}^{2}=$ $\left(\tilde{s}_{i j}\right)_{q \times m}, \tilde{s}_{i j} \in F(R), g, z=1,2, \cdots, K$.

Model IV. A FMMBP-PC problem in which $K(\geq 2)$ followers are involved and there are partial shared decision variables but different objective and constraint functions among them is defined as follows.

For $x \in X \subset R^{n}, y_{i} \in Y_{i} \subset R^{m_{i}}, Y=\left(Y_{1}, \cdots, Y_{k}, Z\right)^{T}$,

$F: X \times Y_{1} \times \times \ldots \times Y_{K} \times Z \rightarrow F\left(R^{s}\right), f_{i}: X \times Y_{i} \times Z \rightarrow$ $F\left(R^{t}\right)$ and $i=1,2 \cdots, K$.

It consists of finding a solution to the upper level problem

$$
\begin{aligned}
& \min _{x \in X} F(x, y)=\left(\tilde{c}_{1}^{1} x+\sum_{j=1}^{K} \tilde{d}_{1 j}^{1} y_{j}+\tilde{d}_{1}^{1} z, \tilde{c}_{2}^{1} x+\sum_{j=1}^{K} \tilde{d}_{2 j}^{1} y_{j}\right. \\
& \left.+\tilde{d}_{2}^{1} z, \cdots, \tilde{c}_{s}^{1} x+\sum_{j=1}^{K} \tilde{d}_{s j}^{1} y_{j}+\tilde{d}_{s}^{1} z\right)^{T} \\
& \text { s.t. } \tilde{A}^{1} x+\sum_{j=1}^{k} \tilde{B}_{j}^{1} y_{j}+\widetilde{B}^{1} z \precsim \tilde{b}^{1}
\end{aligned}
$$

where $y_{i}(i=1,2, \cdots, K)$, for each value of $x$, is the solution of the lower level problem:

$$
\min _{y, \in Y_{J}, z \in Z} f_{i}(x, y)=\left(\tilde{c}_{i 1}^{2} x+\sum_{j=1}^{K} \tilde{d}_{i 1 j}^{2} y_{j}+\tilde{d}_{i 1}^{2} z, \tilde{c}_{i 2}^{2} x+\sum_{j=1}^{K} \tilde{d}_{i 2 j}^{2} y_{j}\right.
$$




$$
\left.+\tilde{d}_{i 2}^{2} z, \cdots, \tilde{c}_{i t}^{2} x+\sum_{j=1}^{K} \tilde{d}_{i j}^{2} y_{j}+\tilde{d}_{i t}^{2} z\right)^{T}
$$

$$
\text { s.t. } \tilde{A}_{r}^{2} x+\sum_{j=1}^{K} \tilde{B}_{r j}^{2} y_{j}+\tilde{B}_{r}^{2} z \supseteqq \tilde{b}_{r}^{2}, r=1,2, \cdots, K \text {, }
$$

where $\quad \tilde{c}_{i}^{1}, \tilde{c}_{z j}^{2} \in F\left(R^{n}\right), \tilde{b}^{1} \in F\left(R^{p}\right), \tilde{b}_{z}^{2} \in F\left(R^{q}\right), \tilde{a}_{i j} \in$ $F(R), \tilde{A}^{1}=\left(\tilde{a}_{i j}\right)_{p \times n}, \tilde{b}_{i j}, \tilde{e}_{i j}^{z} \in F(R), \tilde{B}_{z}^{1}=\left(\tilde{b}_{i j}\right)_{p \times m}, \tilde{A}_{z}^{2}=\left(\tilde{e}_{k j}^{z}\right)_{q \times n}$, $\tilde{d}_{i z}^{1}, \tilde{d}_{g j z}^{2} \in F\left(R^{m}\right), \widetilde{B}_{z j}^{2}=\left(\tilde{s}_{i j}^{z}\right)_{q \times m}, \tilde{s}_{i j}^{z} \in F(R), i=1,2, \cdots, s$, $j=1,2, \cdots, t$, and $g, z=1,2, \cdots, K$.

By analysis above four models and using a weighting method, we can get a general model (Model G) for FMMLB-PC problems:

For $x \in X \subset R^{n}, y_{i} \in Y_{i} \subset R^{m_{i}}, Y=\left(Y_{1}, \cdots, Y_{K}\right)^{T}, F: X \times Y_{1}$ $\times \ldots \times Y_{K} \rightarrow F\left(R^{s}\right), f_{i}: X \times Y_{i} \rightarrow F\left(R^{t}\right)$ and $i=1,2, \ldots, K$.

It consists of finding a solution to the upper level problem

$$
\begin{gathered}
\min _{x \in X} F(x, y)=\left(\tilde{c}_{1}^{1} x+\sum_{j=1}^{K} \tilde{d}_{1 j}^{1} y_{j}, \tilde{c}_{2}^{1} x+\sum_{j=1}^{K} \tilde{d}_{2 j}^{1} y_{j}, \cdots,\right. \\
\left.\tilde{c}_{s}^{1} x+\sum_{j=1}^{K} \tilde{d}_{s j}^{1} y_{j}\right)^{T}
\end{gathered}
$$

$$
\text { s.t. } \tilde{A}^{1} x+\sum_{j=1}^{K} \tilde{B}_{j}^{1} y_{j} \supseteqq \widetilde{b}^{1}
$$

where $y_{i}(i=1,2, \cdots, K)$, for each value of $x$, is the solution of the lower level problem:

$$
\begin{gathered}
\min _{\substack{y \in Y_{Y} \\
j=1,2, \cdots, K}} f(x, y)=\left(\tilde{c}_{1}^{2} x+\sum_{j=1}^{K} \tilde{d}_{1 j}^{2} y_{j}, \tilde{c}_{2}^{2} x+\sum_{j=1}^{K} \tilde{d}_{2 j}^{2} y_{j}, \cdots,\right. \\
\left.\tilde{c}_{t}^{2} x+\sum_{j=1}^{K} \tilde{d}_{i j}^{2} y_{j}\right)^{T}
\end{gathered}
$$

$$
\text { s.t. } \tilde{A}_{z}^{2} x+\sum_{j=1}^{K} \tilde{B}_{z j}^{2} y_{j} \supseteqq \tilde{b}_{z}^{2}, z=1,2, \cdots, K \text {, }
$$

where $\tilde{c}_{i}^{1}, \tilde{c}_{j}^{2} \in F\left(R^{n}\right), \tilde{b}^{1} \in F\left(R^{p}\right), \quad \tilde{b}^{2} \in F\left(R^{q}\right), \quad \tilde{a}_{i j} \in$ $F(R), \quad \tilde{A}^{1}=\left(\tilde{a}_{i j}\right)_{p \times n}, \tilde{b}_{i j} \in F(R), \tilde{B}_{z}^{1}=\left(\tilde{b}_{i j}\right)_{p \times m}, \tilde{e}_{i j} \in F(R), \tilde{d}_{i z}^{1}, \tilde{d}_{j z}^{2}$ $\in F\left(R^{m}\right), i=1,2, \cdots, s, j=1,2, \cdots, t$, and $z=1,2, \cdots, K$, $\tilde{B}_{z}^{2}=\left(\tilde{s}_{i j}\right)_{q \times m}, \tilde{s}_{i j} \in F(R), z=1,2, \cdots, K$.

1. For Models I, II and III, they are obviously special issues of Model IV, respectively.

2. For Model IV, we know that it is a FMMBP problem in which the $K$ followers share the variable $z$. By using weighting method, we can obtain

For $x \in X \subset R^{n}, y_{i} \in Y_{i} \subset R^{m_{i}}, Y=\left(Y_{1}, \cdots, Y_{K}, Y_{K+1}\right)^{T}, F$ : $X \times Y_{1} \times \ldots \times Y_{K} \times Y_{K+1} \rightarrow F\left(R^{s}\right), f_{i}: X \times Y_{i} \rightarrow F\left(R^{t}\right)$ and $i=1,2, \ldots, K+1$.

It consists of finding a solution to the upper level problem

$$
\min _{x \in X} F(x, y)=\left(\tilde{c}_{1}^{1} x+\sum_{j=1}^{K+1} \tilde{d}_{1 j}^{1} y_{j}, \tilde{c}_{2}^{1} x+\sum_{j=1}^{K+1} \tilde{d}_{2 j}^{1} y_{j}, \cdots,\right.
$$

$$
\left.\tilde{c}_{s}^{1} x+\sum_{j=1}^{K+1} \tilde{d}_{s j}^{1} y_{j}\right)^{T}
$$$$
\text { s.t. } \tilde{A}^{1} x+\sum_{j=1}^{K+1} \widetilde{B}_{j}^{1} y_{j} \supseteqq \tilde{b}^{1}
$$

where $y_{i}(i=1,2, \cdots, K)$, for each value of $x$, is the solution of the lower level problem:

$$
\begin{array}{r}
\min _{\substack{y, Y_{J} \\
J=1,2, \cdots, K+1}} f(x, y)=\left(\tilde{c}_{1}^{2} x+\sum_{j=1}^{K+1} \tilde{d}_{1 j}^{2} y_{j}, \tilde{c}_{2}^{2} x+\sum_{j=1}^{K+1} \tilde{d}_{2 j}^{2} y_{j}, \cdots,\right. \\
\left.\tilde{c}_{t}^{2} x+\sum_{j=1}^{K+1} \tilde{d}_{t j}^{2} y_{j}\right)^{T}
\end{array}
$$

s.t. $\tilde{A}_{z}^{2} x+\sum_{j=1}^{K+1} \tilde{B}_{z j}^{2} y_{j} \supseteqq \tilde{b}_{z}^{2}, z=1,2, \cdots, K+1$,

where $\tilde{c}_{i}^{1}, \tilde{c}_{j}^{2} \in F\left(R^{n}\right), \tilde{b}^{1} \in F\left(R^{p}\right), \tilde{b}^{2} \in F\left(R^{q}\right), \quad \tilde{a}_{i j} \in$ $F(R), \tilde{A}^{1}=\left(\tilde{a}_{i j}\right)_{p \times n}, \tilde{b}_{i j} \in F(R), \tilde{B}_{z}^{1}=\left(\tilde{b}_{i j}\right)_{p \times m}, \tilde{e}_{i j} \in F(R)$, $\tilde{d}_{i z}^{1}, \tilde{d}_{j z}^{2} \in F\left(R^{m}\right), \tilde{B}_{z}^{2}=\left(\tilde{s}_{i j}\right)_{q \times m}, \tilde{s}_{i j} \in F(R), i=1,2, \cdots, s$, $j=1,2, \cdots, t$, and $z=1,2, \cdots, K+1$.

3. Obvious, this model and Model $G$ are same.

We will present an approximation branch-andbound algorithm to solve FMMBP problem with in a partial cooperative situation

\section{An Approximation Branch-and- bound Algorithm}

Associated with the FMMBP-PC problem, we now consider the following multi-objective linear bilevel programming (MOLBP) problem:

$$
\begin{aligned}
& \text { For } x \in X \subset R^{n}, y_{i} \in Y_{i} \subset R^{m_{i}}, Y=\left(Y_{1}, \cdots, Y_{K}\right)^{T}, F: X \times Y_{1} \\
& \times \ldots \times Y_{K} \rightarrow F\left(R^{s}\right), f_{i}: X \times Y_{i} \rightarrow F\left(R^{t}\right) \text { and } i=1,2, \ldots, K \text {. } \\
& \min _{x \in X}(F(x, y))_{\lambda}^{L(R)}=\left(\left(F_{1}(x, y)\right)_{\lambda}^{L},\left(F_{1}(x, y)\right)_{\lambda}^{R}, \cdots\left(F_{s}(x, y)\right)_{\lambda}^{L}\right. \text {, } \\
& \left.\left(F_{s}(x, y)\right)_{\lambda}^{R}\right)^{T}, \lambda \in[0,1] \\
& \text { s.t. } A_{\lambda}^{1 L} x+\sum_{j=1}^{K} B_{j \lambda}^{1 L} y_{j} \leqq b_{\lambda}^{1 L} \text {, } \\
& A^{1 R} \stackrel{R}{\lambda} x+\sum_{j=1}^{K} B_{j \lambda}^{1^{R}} y_{j} \leqq b_{\lambda}^{1 R}, \quad \lambda \in[0,1] \\
& \min _{y \in Y}(f(x, y))_{\lambda}^{L(R)}=\left(\left(f_{1}(x, y)\right)_{\lambda}^{L},\left(f_{1}(x, y)\right)_{\lambda}^{R}, \cdots,\right. \\
& \left.\left(f_{t}(x, y)\right)_{\lambda}^{L},\left(f_{t}(x, y)\right)_{\lambda}^{R}\right)^{T}, \lambda \in[0,1] \\
& \text { s.t. } A_{z \lambda}^{2^{L}} x+\sum_{j=1}^{K} B_{z \lambda \lambda}^{2^{L}} y_{j} \leqq b_{z \lambda}^{2^{L}} \text {, } \\
& A_{z \lambda}^{2^{L}} x+\sum_{j=1}^{K} B_{z j \lambda}^{2 L} y_{j} \leqq b_{z \lambda}^{2 L}, \quad z=1,2, \cdots, K, \lambda \in[0,1]
\end{aligned}
$$


$R^{n}, d_{i j \lambda}^{1 L}, d_{i j \lambda}^{1 R}, d_{i j \lambda \lambda}^{2 L}, d_{i j \lambda \lambda}^{2 R} \in R^{m}, b_{\lambda}^{1 L}, b_{\lambda}^{1 R} \in R^{p}, b_{z \lambda}^{2 L}, b_{z \lambda}^{2^{R}} \in R^{q}$,

$A_{\lambda}^{1_{\lambda}^{L}}=\left(a_{i j \lambda}{ }^{L}\right), A_{\lambda}^{1_{\lambda}^{R}}=\left(a_{i j \lambda}{ }^{R}\right) \in R^{p \times n}, A_{z \lambda}^{2^{L}}=\left(e_{i j \lambda \lambda}{ }^{L}\right), A_{z \lambda}^{2^{R}}=\left(e_{i j j_{\lambda}}^{R}\right) \in$

$B_{z j \lambda}^{2^{R}}=R^{q \times n}, B_{z \lambda}^{1^{L}}=\left(b_{i j \lambda \lambda}{ }^{L}\right), B_{z \lambda}^{1^{R}}=\left(b_{i j i \lambda}{ }^{R}\right) \in R^{p \times m}, B_{z j \lambda}^{2^{L}}=\left(s_{i i r \lambda}^{z^{L}}\right)$,

$\left(s_{i r \lambda}^{z i i^{R}}\right) \in R^{q \times m}, i=1,2, \cdots, s, j=1,2, \cdots, t, z=1,2, \cdots, K$.

By using Definition 2.2, we have

Theorem 3.1 Let $\left(x^{*}, y^{*}\right)$ be the optimal solution of the MOLBP problem defined by (2). Then it is also an optimal solution of the FMMBP-PC problem defined by Model G.

We can use the theory of solving FMOLBP [21] to find an optimal solution for Model $G$ because we have known Model $G$ of the general model of FMMBP-PC problem is a FMOLBP problem.

Theorem 3.2 [26] For $x \in X \subset R^{n}, y_{i} \in Y_{i} \subset R^{m_{i}}, i=$ $1,2, \ldots, \mathrm{K}$, if all the fuzzy parameters have piecewise trapezoidal membership functions in the Model G,

$$
\mu_{\tilde{z}}(t)=\left\{\begin{array}{lc}
0 & t<z_{\alpha_{0}}^{L} \\
\frac{\alpha_{1}-\alpha_{0}}{z_{\alpha_{1}}^{L}-z_{\alpha_{0}}^{L}}\left(t-z_{\alpha_{0}}^{L}\right)+\alpha_{0} & z_{\alpha_{0}}^{L} \leqq t<z_{\alpha_{1}}^{L} \\
\frac{\alpha_{1}-\alpha_{0}}{z_{\alpha_{2}}^{L}-z_{\alpha_{1}}^{L}}\left(t-z_{\alpha_{1}}^{L}\right)+\alpha_{1} & z_{\alpha_{1}}^{L} \leqq t<z_{\alpha_{2}}^{L} \\
\cdots & \ldots \\
\alpha & z_{\alpha_{n}}^{L} \leqq t<z_{\alpha_{n}}^{R},(2) \\
\frac{\alpha_{n}-\alpha_{n-1}}{z_{\alpha_{n-1}}^{R}-z_{\alpha_{n}}^{R}}\left(-t+z_{\alpha_{n-1}}^{R}\right)+\alpha_{n-1} & z_{\alpha_{n}}^{R} \leqq t<z_{\alpha_{n-1}}^{R} \\
\frac{\alpha_{0}-\alpha_{1}}{z_{\alpha_{1}}^{R}-z_{\alpha_{0}}^{R}}\left(-t+z_{\alpha_{0}}^{R}\right)+\alpha_{0} & z_{\alpha_{1}}^{R} \leqq t \leq z_{\alpha_{0}}^{R} \\
0 & z_{\alpha_{0}}^{R}<t
\end{array}\right.
$$

where $\tilde{z}$ denotes any fuzzy parameters in Model G, then, $\left(x^{*}, y^{*}\right)$ is a complete optimal solution to the FMMBP-PC problem if and only if $\left(x^{*}, y^{*}\right)$ is an optimal solution to the MOLBP problem:

$$
\begin{aligned}
& \min _{x \in X}\left(F_{i}(x, y)\right)_{\alpha_{j}}^{L}=c_{i \alpha_{j}}^{1^{L}} x+\sum_{z=1}^{K} d_{i z \alpha_{j}}^{1 L} y_{z}, i=1, \ldots, s, j=0,1, \ldots, n \\
& \min _{x \in X}\left(F_{i}(x, y)\right)_{\alpha_{j}}^{R}=c_{i \alpha_{j}}^{1^{R}} x+\sum_{z=1}^{K} d_{i z \alpha_{j}}^{1 R} y_{z}, \\
& \text { s.t. } A_{\alpha_{j}}^{1^{L}} x+\sum_{z=1}^{K} B_{z \alpha_{j}}^{1^{L}} y_{z} \leq b_{\alpha_{j}}^{1^{L}}, j=0,1, \cdots, n \\
& \quad A_{\alpha_{j}}^{1^{R}} x+\sum_{z=1}^{K} B_{z \alpha_{j}}^{1^{R}} y_{z} \leq b_{\alpha_{j}}^{1 R}, j=0,1, \cdots, n \\
& \min _{y \in Y}\left(f_{i}^{z}(x, y)\right)_{\alpha_{j}}^{L}=c_{i \alpha_{j}}^{2^{L}} x+\sum_{k=1}^{K} d_{z i k \alpha_{j}}^{2 L} y_{k}, i=1, \ldots, t, j=0,1, \ldots, n \\
& \min _{y \in Y}\left(f_{i}^{z}(x, y)\right)_{\alpha_{j}}^{R}=c_{i z \alpha_{j}}^{2^{R}} x+\sum_{k=1}^{K} d_{z i k \alpha_{j}}^{2} y_{k}, \quad z=1, \ldots, K, \quad \text { (3) } \\
& \text { s.t. } A_{z \alpha_{j}}^{2^{L}} x+\sum_{z=1}^{K} B_{z z \alpha_{j}}^{2^{L}} y_{z} \leq b_{z \alpha_{j}}^{2^{L}}, j=0,1, \cdots, n \\
& A_{z \alpha_{j}}^{2^{R}} x+\sum_{z=1}^{K} B_{z z \alpha_{j}}^{2^{R}} y_{z} \leqq b_{z \alpha_{j}}^{2^{R}}, \quad z=1,2, \cdots, K
\end{aligned}
$$

We note

$$
\begin{aligned}
& \bar{A}_{1} x+\bar{B}_{1} y<\bar{b}_{1} \\
& \bar{A}_{2} x+\bar{B}_{2} y \_\bar{b}_{2}
\end{aligned}
$$

where

$$
\begin{aligned}
& \bar{A}_{1}=\left(\begin{array}{c}
A_{\alpha_{\alpha_{0}}}^{1^{2}} \\
\vdots \\
A_{\alpha_{n}}^{1^{L}} \\
A_{\alpha_{0}}^{1^{R}} \\
\vdots \\
A_{\alpha_{\alpha_{n}}}^{1^{R}}
\end{array}\right), \bar{A}_{2}=\left(\begin{array}{c}
A_{1 \alpha_{0}}^{2^{L}} \\
\vdots \\
A_{K \alpha_{n}}^{2^{L}} \\
A_{1 \alpha_{0}}^{2^{R}} \\
\vdots \\
A_{K \alpha_{n}}^{2^{R}}
\end{array}\right), \bar{B}_{1}=\left(\begin{array}{c}
\sum_{z=1}^{K} B_{z \alpha_{0}}^{1^{L}} \\
\vdots \\
\sum_{z=1}^{K} B_{z \alpha_{n}}^{1^{L}} \\
\sum_{z=1}^{K} B_{z \alpha_{0}}^{1^{R}} \\
\vdots \\
\sum_{z=1}^{K} B_{z \alpha_{n}}^{1^{R}}
\end{array}\right), \\
& \bar{B}_{2}=\left(\begin{array}{c}
\sum_{z=1}^{K} B_{z 1 \alpha_{0}}^{2^{L}} \\
\vdots \\
\sum_{z=1}^{K} B_{z K \alpha_{n}}^{2 L} \\
\sum_{z=1}^{K} B_{z 1 \alpha_{0}}^{2^{2 R}} \\
\vdots \\
\sum_{z=1}^{K} B_{z K \alpha_{n}}^{2^{R}}
\end{array}\right), \bar{b}_{1}=\left(\begin{array}{c}
b^{1 L} \\
\alpha_{0} \\
\vdots \\
b^{1 L} \alpha_{n} \\
b^{1 \alpha_{0}} \\
\vdots \\
b^{1 \alpha_{n}}
\end{array}\right), \bar{b}_{2}=\left(\begin{array}{c}
b_{1 \alpha_{0}}^{2^{L}} \\
\vdots \\
b_{K \alpha_{n}}^{2^{L}} \\
b_{1 \alpha_{0}}^{2^{R}} \\
\vdots \\
b_{K \alpha_{n}}^{2^{R}}
\end{array}\right) .
\end{aligned}
$$

Then we can re-write (3) by using

$$
\begin{aligned}
& \min _{x \in X}\left(F_{i}(x, y)\right)_{\alpha_{j}}^{L}=c_{i \alpha_{j}}^{1 L} x+\sum_{z=1}^{K} d_{i z \alpha_{j}}^{1 L} y_{z}, i=1, \ldots, s, j=0,1, \ldots, n \\
& \min _{x \in X}\left(F_{i}(x, y)\right)_{\alpha_{j}}^{R}=c_{i \alpha_{j}}^{1^{R}} x+\sum_{z=1}^{K} d_{i z \alpha_{j}}^{1^{R}} y_{z} \text {, } \\
& \text { s.t. } \bar{A}_{1} x+\bar{B}_{1} y \leq \bar{b}_{1} \text {, } \\
& \min _{y \in Y}\left(f_{i}^{z}(x, y)\right)_{\alpha_{j}}^{L}=c_{i k \alpha_{j}}^{2^{L}} x+\sum_{k=1}^{K} d_{z i k \alpha_{j}}^{2 L} y_{k}, \quad i=1, \ldots, t, j=0,1, \ldots, n \\
& \min _{y \in Y}\left(f_{i}^{z}(x, y)\right)_{\alpha_{j}}^{L}=c_{i z \alpha_{j}}^{2^{L}} x+\sum_{k=1}^{K} d_{z i k \alpha_{j}}^{2 L} y_{k}, i=1, \ldots, t, j=0,1, \ldots, n \\
& \min _{y \in Y}\left(f_{i}^{z}(x, y)\right)_{\alpha_{j}}^{R}=c_{i z \alpha_{j}}^{2^{R}} x+\sum_{k=1}^{K} d_{z i k \alpha_{j}}^{2 R} y_{k}, \quad z=1, \ldots, K, \quad \text { (3'C) } \\
& \text { s.t. } \bar{A}_{2} x+\bar{B}_{2} y \leq \bar{b}_{2} \text {. }
\end{aligned}
$$

Theorem 3.3 [26] For $x \in X \subset R^{n}, y_{i} \in Y_{i} \subset R^{m_{i}}, i=$ $1,2, \ldots, K$, if all the fuzzy parameters have piecewise trapezoidal membership functions in the Model G, then a necessary and sufficient condition that $\left(x^{*}, y^{*}\right)$ solves the Model $\mathrm{G}$ problem is that there exist vectors $u^{*}, v^{*}$ and $z^{*}$ such that $\left(x^{*}, y^{*}, u^{*}, v^{*}, z^{*}\right)$ solves:

$$
\begin{aligned}
& \min _{x \in X}(F(x, y))=\sum_{j=1}^{s} w_{j}^{1}\left(\sum_{i=0}^{n}\left(c_{j \alpha_{\alpha_{j}}}^{L} x+\sum_{z=1}^{K} d_{i z \alpha_{j}} y_{z} y_{z}\right)\right. \\
& \left.+\quad+\sum_{i=0}^{n}\left(c_{j 1 \alpha_{i}}^{R} x+\sum_{z=1}^{K} d_{i z \alpha_{j}}{ }^{1 R} y_{z}\right)\right) \\
& \text { s.t. } \bar{A}_{1} x+\bar{B}_{1} y \leq \bar{b}_{1}
\end{aligned}
$$




$$
\begin{gathered}
\bar{A}_{2} x+\bar{B}_{2} y \leq \bar{b}_{2} \\
u\left(\sum_{i=0}^{n} \sum_{z=1}^{K} B_{z \alpha_{i}}^{1^{L}}+\sum_{i=0}^{n} \sum_{z=1}^{K} B_{z \alpha_{i}}^{1^{R}}\right)+v\left(\sum_{i=0}^{n} \sum_{z=1}^{K} \sum_{j=1}^{K} B_{z j \alpha_{i}}^{2^{L}}+\sum_{i=0}^{n} \sum_{z=1}^{K} \sum_{j=1}^{K} B_{z j \alpha_{i}}^{2^{R}}\right) \\
-z=-\sum_{j=1}^{t} w_{j}^{2}\left(\sum_{i=0}^{n} \sum_{z=1}^{K} d_{z j \alpha_{i}}^{2^{L}}+\sum_{i=0}^{n} \sum_{z=1}^{K} d_{z j}^{2^{R}}\right) \\
u\left(\left(\sum_{i=0}^{n} b_{\alpha_{i}}^{1^{L}}+\sum_{i=0}^{n} b_{\alpha_{i}}^{1^{R}}\right)-\left(\sum_{i=0}^{n} A_{\alpha_{i}}^{1^{L}}+\sum_{i=0}^{n} A_{\alpha_{i}}^{1^{R}}\right) x-\left(\sum_{i=0}^{n} \sum_{z=1}^{K} B_{z \alpha_{i}}^{1^{L}}+\right.\right. \\
\left.\left.\sum_{i=0}^{n} \sum_{z=1}^{K} B_{z \alpha_{i}}^{1^{R}}\right) y\right)++v\left(\left(\sum_{i=0}^{n} \sum_{j=1}^{K} b_{j}^{2^{L} \alpha_{i}}+\sum_{i=0}^{n} \sum_{j=1}^{K} b_{j}^{2} \alpha_{\alpha_{i}}\right)-\right. \\
\left(\sum_{i=0}^{n} \sum_{j=1}^{K} A_{j \alpha_{i}}^{2^{L}}+\sum_{i=0}^{n} \sum_{j=1}^{K} A_{j \alpha_{i}}^{2^{R}}\right) x-\left(\sum_{i=0}^{n} \sum_{z=1}^{K} \sum_{j=1}^{K} B_{z j \alpha_{i}}^{2^{L}}+\right. \\
\left.\left.\sum_{i=0}^{n} \sum_{z=1}^{K} \sum_{j=1}^{K} B_{z j \alpha_{i}}^{2^{R}}\right) y\right)+z y=0 \\
x \geq 0, y \geq 0, u \geq 0, v \geq 0, z \geq 0 \\
\text { where } \sum_{j=1}^{s} w_{j}^{1}=1 \text { and } \sum_{j=1}^{t} w_{j}^{2}=1 .
\end{gathered}
$$

Based on Theorem 3.3, we will present an approximation branch-and-bound algorithm for solving the FMMBP-PC problem shown in Model G.

We first write all the inequalities (except of the leader's variables) of (3'a)-(3'd) as $g_{i}(x, y) \geq 0, i=1$, $2, \ldots, p+q+m$, and note that complementary slackness simply means $u_{i} g_{i}(x, y)=0(i=1,2, \ldots$, $p+q+m)$. Now we suppress the complementary term and solve the resulted linear sub-problem. At each time of iteration the condition (4e) is checked. If it is satisfied, the corresponding point is in the inducible region and hence a potential solution to (3'). Otherwise, a branch-and-bound scheme is used to implicitly examine all combinations of the complementarities slackness.

Let $W=\{1,2, \ldots, p+q+m\}$ be the index set for the terms in (4e), $\bar{F}$ be the incumbent upper bound on the objective function of the leader. At the $k$ th level of an search tree we define a subset of indices $W_{k} \subset W$, and a path $P_{k}$ corresponding to an assignment of either $u_{i}=0$ or $g_{i}=0$ for $i \in W_{k}$. Now let

$$
\begin{aligned}
& S_{k}^{+}=\left\{i: i \in W_{k}, u_{i}=0\right\} \\
& S_{k}^{-}=\left\{i: i \in W_{k}, g_{i}=0\right\} \\
& S_{k}^{0}=\left\{i: i \notin W_{k}\right\} .
\end{aligned}
$$

For $i \in S_{k}^{0}$, the variables $u_{i}$ or $g_{i}$ are free to assume any nonnegative value in the solution of (4) with (4e) omitted, so complementary slackness will not necessarily be satisfied.

By using these notations we give all steps of the approximation branch-and-bound algorithm for the proposed FMMBP-PC problem:
Step 1. Give weights for the objectives of the leader and the followers, and let $\sum_{j=1}^{s} w_{j 1}=1$ and $\sum_{j=1}^{t} w_{j 2}=1$.

Step 2. Transform Model G to the problem (3’)

Step 3. Let the interval $[0,1]$ be decomposed into $2^{l-1}$ mean sub-intervals with $\left(2^{l-1}+1\right)$ nodes $\lambda_{i}\left(i=0, \cdots, 2^{l-1}\right)$ which are arranged in the order of $0=\lambda_{0}<\lambda_{1}<\cdots<\lambda_{2^{l-1}}=1$ and a range of errors $\varepsilon>0$.

Step 4. Set $l=1$, then solve (MOLBP) ${ }_{2}{ }_{2}$, i.e. (3') by using an extended branch-and-bound algorithm [15] when $\beta=0$ and $\alpha=1$, we obtain an optimization solution $(x, y)_{2^{l}}$.

Step 5. Transform the problem (3') to linear BP problem (4) by using a weighting method [10].

Step 6. Solve the problem (4).

Step 7. (Initialization) Set $k=0, S_{k}^{+}=\phi, S_{k}^{-}=\phi$, $S_{k}^{0}=\{1, \ldots, p+q+m\}$, and $\bar{F}=\infty$.

Step 8. (Iteration $k$ ) Set $u_{i}=0$ for $i \in S_{k}^{+}$and $g_{i}=0$ for $i \in S_{k}^{-}$. It first attempts to solve (4) without (4e). If the resultant problem is infeasible, go to Step 12; otherwise, put $k \leftarrow k+1$ and label the solution $\left(x^{k}, y^{k}, u^{k}\right)$.

Step 9. (Fathoming) If $F\left(x^{k}, y^{k}\right) \geq \bar{F}$, then go to Step 12.

Step 10. (Branching) If $u_{i}^{k} g_{i}\left(x^{k}, y^{k}\right)=0, i=1$, $2, \ldots, p+q+m$, then go to Step 11. Otherwise select $i$ for which $u_{i}^{k} g_{i}\left(x^{k}, y^{k}\right) \neq 0$ is the largest and label it $i_{1}$. Put $S_{k}^{+} \leftarrow S_{k}^{+} \cup\left\{i_{1}\right\}, S_{k}^{0} \leftarrow S_{k}^{0} \backslash\left\{i_{1}\right\}, S_{k}^{-} \leftarrow S_{k}^{-}$, append $i_{1}$ to $P_{k}$, and go to Step 8 .

Step 11. (Updating) Let $\bar{F} \leftarrow F\left(x^{k}, y^{k}\right)$.

Step 12. (Backtracking) If no live node exists, go to Step 13. Otherwise branch to the newest live vertex and update $S_{k}^{+}, S_{k}^{-}, S_{k}^{0}$ and $P_{k}$ as discussed below. Go back to Step 8 .

Step 13. (Termination) If $\bar{F}=\infty$, there is no feasible solution to (MOLBP) ${ }_{2}^{l}$. Otherwise, declare the feasible point associated with $\bar{F}$ which is the optimal solution to (MOLBP) $)_{2}^{l}$.

Step 14. Solve (MOLBP) ${ }_{2}^{l+1}$ by Step 8 to Step 13, and we obtain an optimization solution $(x, y)_{2^{t+1}}$. 
Step 15. If $\left\|(x, y)_{2^{t+1}}-(x, y)_{2^{2}}\right\|<\varepsilon$, then the solution $\left(x^{*}, y^{*}\right)$ of the FMMBP-PC problem is $(x, y)_{2^{++1}}$. Otherwise, update $l$ to $2 l$ and go back to Step 8 .

Step 16. Show the result of FMMBP-PC problem.

We give some explanations for these steps and their working process as follows.

After initialization, Step 8 is designed to find a new point which is potentially bilevel feasible. If no solution exists, or the solution does not offer an improvement over the incumbent (Step 9), the algorithm goes to Step 12 and backtracks.

Step 10 checks the value of $u_{i}^{k} g_{i}\left(x^{k}, y^{k}\right)$ to determine if the complementary slackness conditions are satisfied. In practice, if $\left|u_{i}^{k} g_{i}\right|<10^{-6}$ it is considered to be zero. Confirmation indicates that a feasible solution of a FMMBP-PC has been found and at Step 11 the upper bound on the leader's objective function is updated. Alternatively, if the complementary slackness conditions are not satisfied, the term with the largest product is used at Step 10 to provide a branching variable. Branching is always completed on the Kuhn-Tucker multiplier [2].

At Step 12, the backtracking operation is performed. Note that a live node is one associated with a sub-problem that has not yet been fathomed at either Step 8 due to infeasibility or at Step 9 due to bounding, and whose solution violates at least one complementary slackness condition. To facilitate book keeping, the path $P_{k}$ in the branch-and-bound tree is represented by a vector, its dimension is the current depth of the tree. The order of the components of $P_{k}$ is determined by their level in the tree. Indices only appear in $P_{k}$ if they are in either $S_{k}^{+}$or $S_{k}^{-}$with the entries underlined if they are in $S_{k}^{-}$. Because the algorithm always branches on a Kuhn-Tucker multiplier first, backtracking is accomplished by finding the rightmost non-underlined component if $P_{k}$, underlining it, and erasing all entries to the right. The erased entries are deleted from $S_{k}^{-}$and added to $S_{k}^{0}$.

\section{Conclusions}

A bilevel decision making problem may be modeled to have multiple objective functions, fuzzy parameters, and multiple followers. The research deals with the three issues together. This paper proposes a fuzzy number based approximation branch-and-bound algorithm to solve this complex problem, i.e., fuzzy multi-objective multi-follower linear bilievel problem with a partial decision variable among followers. Further study includes the development of models and approaches for other situation among followers: when followers do not share their decision variables.

\section{Acknowledgment}

The work presented in this paper was supported by Australian Research Council (ARC) under discovery grant DP0557154.

\section{References}

[1] G. Anandalingam and T. Friesz, Hierarchical optimization: An introduction, Annals of Operations Research 34:1-11, 1992.

[2] J. Bard, Practical bilevel optimization: Algorithms and applications, Amsterdam: Kluwer Academic Publishers, 1998.

[3] W. Bialas and M. Karwan, Two-level linear programming, Management Science 30:10041020, 1984.

[4] J. Bracken and J. McGill, Mathematical programs with optimization problems in the constraints, Operations Research 21: 37-44, 1973.

[5] W. Candler and R. Townsley, A linear two-level programming problem, Computers and Operations Research 9 : 59-76, 1982.

[6] S. Dempe, A simple algorithm for the linear bilevel programming problem, Optimization 18:373-385, 1987.

[7] P. Hansen, B. Jaumard, and G. Savard, New branch-and-bound rules for linear bilevel programming, SIAM Journal on Scientific and Statistical Computing 13:1194-1217, 1992.

[8] J. Lu, C. Shi, and G. Zhang, An extended branchand-bound algorithm for bilevel multi-follower decision making in a referential-uncooperative situation, International Journal of Information Technology and Decision Making 6:1-18, 2007.

[9] J. Lu, C. Shi and G. Zhang, On bilevel multifollower decision-making: general framework and solutions, Information Science 176:16071627, 2006.

[10] J. Lu, G. Zhang, D. Ruan and F. Wu, Multiobjective group decision making: methods, software and applications with fuzzy set technology, Imperial College Press, London, 2007.

[11] J. Lu, G. Zhang and T. Dillon, Fuzzy multiobjective bilevel decision making by an approximation Kth-best approach, Journal of Multiple-Valued Logic and Soft Computing, in press.

[12] T. Miller, T. Friesz and R. Tobin, Heuristic algorithms for delivered price spatially competitive network facility location problems, Annals of Operations Research 34:177-202, 1992.

[13] M. Sakawa, Fussy sets and interactive multiobjective optimization, New York: Plenum Press, 1993.

[14] M. Sakawa, I. Nishizaki, and Y. Uemura, Interactive fuzzy programming for multilevel 
linear programming problems with fuzzy parameters, Fuzzy Sets and Systems 109: 3-19, 2000.

[15] C. Shi, J. Lu and G. Zhang, An extended KuhnTucker approach for linear bilevel programming, Applied Mathematics and Computation 162:5163, 2005.

[16] C. Shi, J. Lu and G. Zhang, An extended Kthbast approach for linear bilevel programming, Applied Mathematics and Computation 164: 843855, 2004.

[17] C. Shi, G. Zhang and J. Lu, On the definition of linear bilevel programming solution, Applied Mathematics and Computation 160: 169-176, 2005.

[18] H. Von Stackelberg, The theory of the market economy, New York: Oxford University Press, 1952.

[19] D. White and G. Anandalingam, “A penalty function approach for solving bi-level linear programs," Journal of Global Optimization, 3:397-419, 1993.

[20] L. A Zadeh, Fuzzy sets, Information \& Control 8:338-353, 1965.

[21] G. Zhang and J. Lu, The definition of optimal solution and an extended Kuhn-Tucker approach for fuzzy linear bilevel programming, IEEE Computational Intelligence Bulletin, 5:1-7, 2005.

[22] G. Zhang and J. Lu, Model and approach of fuzzy bilevel decision making for logistics planning problem, Journal of Enterprise Information Management 20:178-197, 2007.

[23] G. Zhang, J. Lu and T. Dillon, A branch-andbound algorithm for fuzzy bilevel decision making, in: Ruan, et al eds., Applied Artificial Intelligence, Singapore : World Scientific, pp. 291-298, 2006.

[24] G. Zhang, J. Lu and T. Dillon, Kth-best algorithm for fuzzy bilevel programming, International Conference on Intelligent Systems and Knowledge Engineering (ISKE2006), pp.6-7, Shanghai, China (CDRoom), 2006.

[25] G. Zhang, J. Lu and T. Dillon, An approximation branch-and-bound approach for fuzzy linear bilevel decision making, $1^{\text {st }}$ International Symposium Advances in Artificial Intelligence and Applications (AAIA `06) Wisla, Poland, November 6-10, 2006 (CDRoom).

[26] G. Zhang, J. Lu and T. Dillon, Solution concepts and an approximation Kuhn-Tucker approach for fuzzy multi-objective linear bilevel programming, in: Pardalos et al eds., Pareto Optimality, Game Theory and Equilibria: Spinger, pp. 467-490, 2007

[27] G. Zhang, J. Lu and T. Dillon, Decentralized multi-objective bilevel decision making with fuzzy demands, Knowledge-Based System 20:495-507, 2007.

[28] G. Zhang, J. Lu and T. Dillon, Models and algorithm for fuzzy multi-objective multifollower linear bilevel programming, IEEE International Conference on Fuzzy Systems, Imperial College, London, UK,pp. 23-26, 2007. 